\title{
Dispersive pressure and density variations in snow avalanches
}

\author{
Othmar BUSER, Perry BARTELT
}

\begin{abstract}
WSL Swiss Federal Institute for Snow and Avalanche Research SLF, Flüelastrasse 11, CH-7260 Davos-Dorf, Switzerland E-mail: bartelt@slf.ch
\end{abstract}

\begin{abstract}
Snow avalanches possess two types of kinetic energy: the kinetic energy associated with the mean velocity in the downhill direction and the kinetic energy associated with individual particle velocities that vary from the mean. The mean kinetic energy is directional; the kinetic energy associated with the velocity fluctuations is non-directional in the sense that it is connected to random particle movements. However, the rigid, basal boundary directs the random fluctuation energy into the avalanche. Thus, the random energy flux is converted to free mechanical energy which lifts and dilates the avalanche flow mass, changing the flow density and increasing the normal (dispersive) pressure and, as a consequence, changing the flow resistance. In this paper we derive macroscopic relations that link the production of the random kinetic energy to the perpendicular acceleration of the avalanche's center of mass. We show that a single burst of fluctuation energy will produce pressures that oscillate around the hydrostatic pressure. Because we do not include a damping process, the oscillations of the center of mass remain, even if the production of random kinetic energy stops. We formulate relationships that can be used within the framework of depth-averaged mass and momentum equations that are often used to simulate snow avalanches in realistic terrain.
\end{abstract}

\section{INTRODUCTION}

A fundamental problem in natural hazard research is to understand how velocity fluctuations in fast-moving, gravitationally driven slides of snow, rock or earthen debris produce a dispersive pressure that fluidizes the moving mass, increasing mobility and danger (Issler and Gauer, 2008; Bartelt and Buser, 2011). Dispersive pressure (Bagnold, 1954) arises in dense snow avalanches and other dry, granular-type debris flows because of shear deformations in the granular material, which consists of particles (snow clods, rocks, earth clumps, woody debris and other detritus). As the flow shears, collisions between particles produce accelerations of the granular fluid perpendicular to the flow direction. The pressure associated with the dilatative strains (the dispersive pressure, $p$ ) raises the center of mass of the avalanche, changing the normal pressure, $N$, and therefore the shear resistance, $S$, of the flow on the ground.

The primary difficulty in the study of dispersive pressure is to define an appropriate reference volume where we can average the outcome of the particle collisions, the source of $p$. Unlike Bagnold, who considered the dispersive pressure in a constant volume, we consider a volume with a free boundary, the top surface of the avalanche. Since we cannot assume that all the particles have identical velocity vectors (magnitude and direction), there must be some variation about the mean. With this variation there is an associated kinetic energy, that we term random. By summing the random particle energies in a welldefined reference volume we can find the density of the random kinetic energy, $R$. In this paper we relate temporal changes of $R$ to the perpendicular acceleration of the center of mass of the reference volume. The change of $R$ can be abrupt, as when the avalanche runs over rough ground. To model how these bursts of $R$ affect the dispersive pressure we do not calculate the trajectory of each individual particle by integrating well-known equations of motion, including momentum exchanges arising from binary collisions. Instead, by developing a macroscopic model for dispersive pressure in an open volume, we find a strong resemblance to the thermodynamic gas law; that is, density and pressure changes are related to a change of the random kinetic energy, known as the 'granular temperature'.

However, by formulating a macroscopic model we lose information concerning the individual particle trajectories and, therefore, density distributions and any other microscopic feature. At present, microscopic features of the flow are extremely difficult to measure in real avalanches. Our measurement of dispersive pressure necessarily involves ensembles of many particle interactions that are true only on average. Individual particle impact pressures at the bottom will be higher than the mean $p$, but it is this mean, macroscopic $p$ that we measure (Platzer and others, 2007). As the center of mass of the reference volume is also defined by the ensemble of the individual particles, calculating bulk changes in the perpendicular direction is not only a mechanical consequence of the changes in the mean $p$, but also results in a consistent formulation for continuum avalanche flow models (Gubler, 1987; Norem and others, 1987; Salm, 1993; Naaim and others, 2004; Christen and others, 2010). To study the role of dispersive pressure in avalanches, we must therefore start by carefully defining our reference volume and the energy density, $R$.

\section{RANDOM KINETIC ENERGY}

We consider a two-dimensional flow segment of an avalanche filled with granular material (snow clods, rocks, woody debris; Bartelt and McArdell, 2009), as shown in Figure 1 . The space between the snow clods is filled with air and contains individual snow grains or ice crystals freed by the abrasive interaction between larger snow clumps. Because the mass of snow grains is small in comparison with the mass of large debris, we consider the snow grains to be part of the air phase. The flow segment extends from the base of the avalanche, the running surface, to the top surface. The base has unit area and therefore the flow volume is defined by the flow height, $h_{0}$. Because we assume an isotropic (homogeneous) density, $\rho_{0}$, the mass per unit area of the 


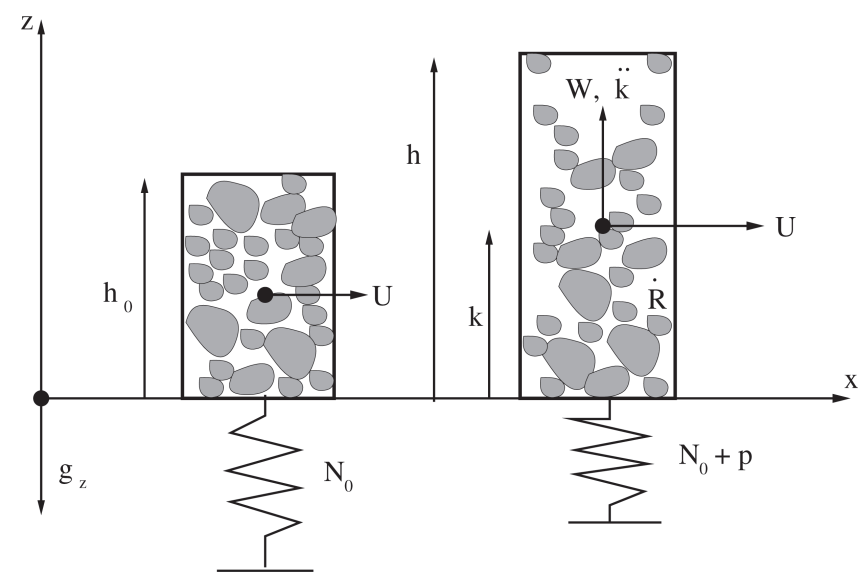

Fig. 1. Segment of a flowing avalanche with height $h_{0}$ and homogeneous density $\rho_{0}$. The normal pressure on the bottom is $N_{0}=\rho_{0} g_{z} h_{0}$. Random kinetic energy is introduced into the segment at the rate $\dot{\mathbf{R}}$. The flow height increases from $h_{0}$ to $h$. The center of mass moves with velocity $\mathbf{W}$. The measured pressure on the bottom will be $N=N_{0}+p$, where $p$ is the dispersive pressure.

segment is $m_{0}=\rho_{0} h_{0}$. We term the homogeneous density, $\rho_{0}$, the reference density of the flow because it is associated with the reference volume, $V_{0}$. The flow segment moves in the $x$-direction with mean velocity $\mathbf{U}$. If a burst of fluctuation energy occurs, the flow height increases from $h_{0}$ to $h$ and the density decreases from $\rho_{0}$ to $\rho$ (Fig. 1). Although it is an arbitrary choice, we usually take the deposition density for the reference density, $\rho_{0}$. The flow density, $\rho$, of the segment will in general differ from the reference density.

Because there is no interaction with neighboring segments or mass flux through the lower and/or upper segment boundaries, the mass remains constant and therefore the relationship $m_{0}=\rho_{0} h_{0}=\rho h$ holds (Fig. 1). The assumption of homogeneous density implies that some of the solid material is in contact with the boundaries (bottom, sides and top), corresponding to the perimeter of the closed volume.

The mean velocities parallel and perpendicular to the slope, $\mathbf{U}$ and $\mathbf{W}$, respectively, are found by averaging the $n$ granule velocities $\left(U_{i}, W_{i}\right)$ in the segment in the $x$ - and z-directions:

$$
\mathbf{U}=\frac{1}{n} \sum_{i=1}^{n} U_{i}, \quad \mathbf{W}=\frac{1}{n} \sum_{i=1}^{n} W_{i}
$$

The velocity fluctuation of the $i$ th granule can then be found,

$$
u_{i}=U_{i}-\mathbf{U}, \quad w_{i}=W_{i}-\mathbf{W} .
$$

These fluctuation velocities define the random kinetic energy density, $\mathbf{R}$, in the segment

$$
\mathbf{R}=\frac{1}{2 h_{0}} \sum_{i=1}^{n} m_{i}\left(u_{i}^{2}+w_{i}^{2}\right),
$$

where $m_{i}$ is the mass of the $i$ th granule. Dividing by the segment volume, $h_{0}$, and the unit basal area $\left(1 \mathrm{~m}^{2}\right)$ we find the mean specific energy. Thus, as the avalanche segment moves in the downslope direction, it experiences changes in both $\mathbf{U}$ and $\mathbf{R}$. In general, the energy, $\mathbf{R}$, is random in nature and therefore $\mathbf{R}$ cannot perform mechanical work. However, at the base of the avalanche a flux of $\mathbf{R}$ is deflected by the running surface upwards into the segment (Fig. 1). The granular burst is given by the flux, $\dot{\mathbf{R}}$. This energy flux raises the center of mass, converting a random energy flux into potential energy (it performs mechanical work). The rise in the center of mass is associated with the dispersive pressure, $p$. At present it is not necessary to know the exact nature of the sources of random kinetic energy, $\dot{\mathbf{R}}$. It can arise from basal shearing (Salm, 1993), i.e. sudden changes in surface roughness and slope, or it can arise from internal shearing movements between granules in a near-boundary slip volume (Bartelt and others, 2006; Buser and Bartelt, 2009).

\section{DISPERSIVE PRESSURE}

The variable $h$ is the height of the avalanche and is associated with the avalanche's center of mass, $k=h / 2$ (homogeneous density distribution). Thus, $\mathbf{W}=\dot{k}$. The center of mass moves in the $z$-direction. No friction, no bending, no shear motion or any other form of damping is considered. For simplicity, we assume only a homogeneous density distribution. By denoting the $z$-location of the center of mass, $k$, we underscore the importance of tracking the vertical velocity, acceleration and change of acceleration of the center of mass. Before we apply a granular burst of $\mathbf{R}$, we assume that the mean velocity in the perpendicular $z$-direction (the velocity of the center of mass) is zero, $\mathbf{W}=0$, or at least known.

The normal stress, $N$, acting on the ground is

$$
N=N_{0}+p=\rho_{0} h_{0}\left(g_{z}+\ddot{k}\right),
$$

where $N_{0}=m_{0} g_{z}=\rho_{0} h_{0} g_{z}$ is due to the static weight of the avalanche; $g_{z}$ is the $z$-component of the gravitational acceleration. The pressure, $p=\rho_{0} h_{0} \ddot{k}$, is the inertial force per unit area associated with the acceleration of the center of mass, $\ddot{k}$. This is the dispersive pressure (or excess pressure or reaction) that would be measured on the ground due to accelerating the center of mass upwards. To measure $N$ we use a force plate that is sufficiently large, such that we do not measure individual impacts. The time rate of change of $N$ is

$$
\dot{N}=\dot{p}=\rho_{0} h_{0} \dddot{k} \text {. }
$$

This equation assumes that the mass, $\rho_{0} h_{0}$, is constant during the time interval over which we convert $\dot{\mathbf{R}}$ to mechanical work on the bottom boundary. In experiments where the normal pressure is measured, the pressure, $p$, induced by the acceleration, $\ddot{k}$, cannot be discerned from an increase in mass. Only by tracking the location of the center of mass, $k$, in time can we decide how the random kinetic energy influences the dispersive pressure. The dispersive pressure cannot act continuously in time because this would mean a constant $\ddot{k}$, which implies a steady, ever-increasing height (or, if it decreases, an infinite density, $\rho$ ). This is clearly not the case. The normal force, $N=N_{0}+p$, fluctuates around $N_{0}$. This is why we have to calculate changes in $\ddot{k}$.

We can attribute the change in random kinetic energy to the change in inner energy, $N h$, and from this we find the relationship between $\mathbf{R}$ and $h$ by solving

$$
\frac{\mathrm{d}}{\mathrm{d} t}(\mathrm{~N} h)=\dot{\mathrm{N}} h+\dot{h} \mathrm{~N}=\gamma \dot{\mathbf{R}} h
$$

This equation relates the change of inner energy, $\mathbf{R}$, to the change in pressure, $N$, and volume, $h$. It resembles the ideal gas law, $p V \propto$ thermal temperature. Thus we assume that a change in random kinetic energy corresponds to the change in thermal energy in kinetic gas theory. In Equation (6), the burst of $\mathbf{R}$ increases the inner energy and the potential 

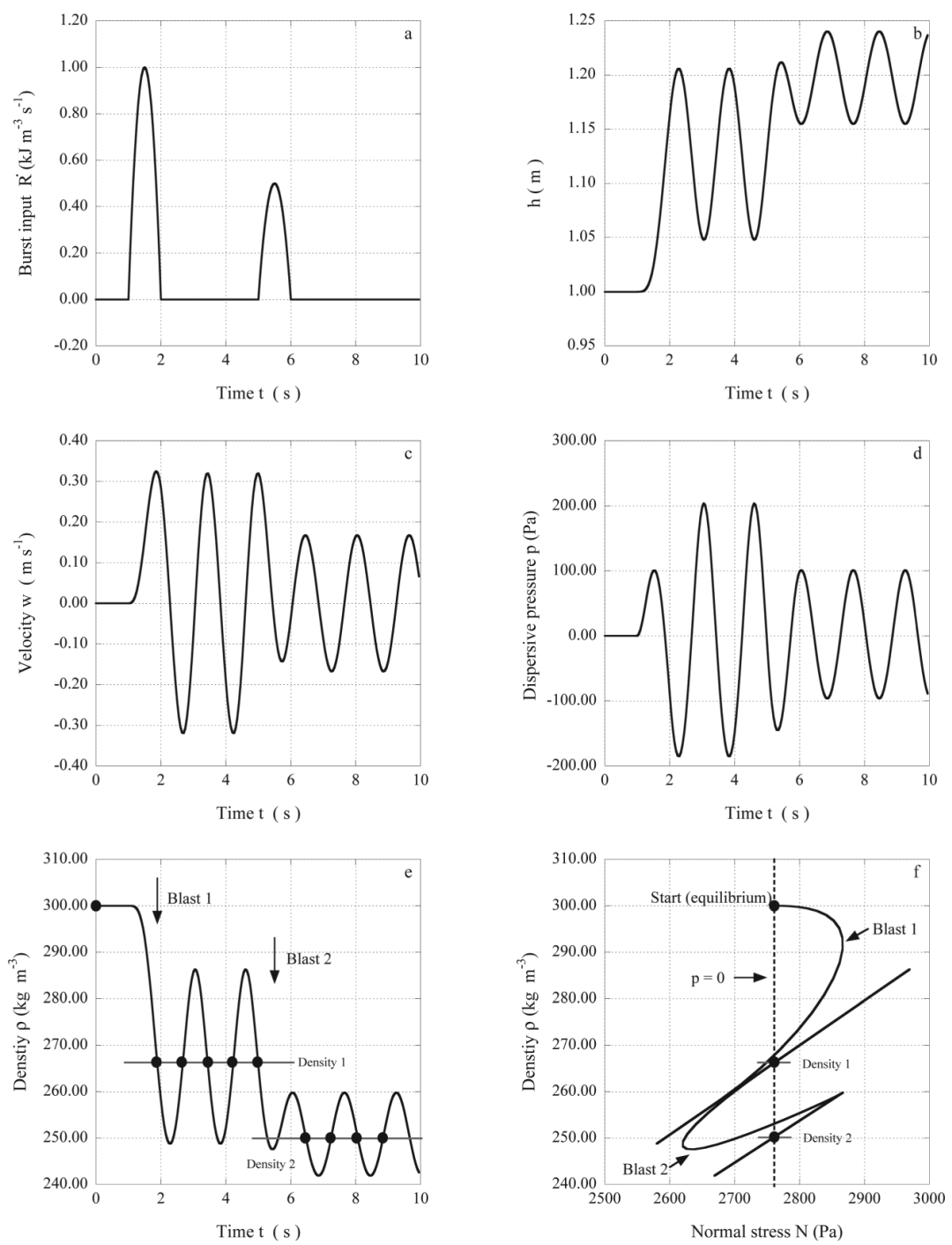

Fig. 2. Two bursts of random kinetic energy are applied to an avalanche with initial flow height, $h_{0}=1 \mathrm{~m}$, and flow density, $\rho=300 \mathrm{~kg} \mathrm{~m}{ }^{-3}$. (a) Random kinetic energy input, $\dot{\mathbf{R}}$. Two bursts of random energy. (b) Calculated height, $h$, the true height of the avalanche. (c) Vertical velocity of the center of mass, W. (d) Dispersive pressure, $p$. (e) Calculated flow density, $\rho$. After each burst the density decreases and oscillates around a new level. In this case, $\rho=265 \mathrm{~kg} \mathrm{~m}^{-3}$ after burst 1 and $\rho=250 \mathrm{~kg} \mathrm{~m}^{-3}$ after burst 2. (f) Flow density and normal pressure. The system starts in equilibrium $N_{0}$. Because the mass does not change, the system oscillates around $N_{0}$ where $p=0$.

energy such that energy is conserved $(\gamma=1)$. Therefore, the coefficient, $\gamma$, describes the efficiency of this process. It can be defined, for example, to account for mass leaks, or sideways transport of $\mathbf{R}, \gamma \neq 1$. Since $\mathbf{R}$ is the random energy density, we multiply by $h_{0}$ to find the mean random kinetic energy per square meter. Clearly, the coefficient, $\gamma$, will change, depending on the distribution of $\mathbf{R}$ in the vertical direction. Substituting the equations for $p$ (Equation (4)) and $\dot{p}$ (Equation (5)) into the change-of-energy equation (Equation (6)) leads to a third-order differential equation in $h$ :

$$
\frac{\dddot{h}}{2}+\left(g_{z}+\frac{\ddot{h}}{2}\right) \frac{\dot{h}}{h}=\frac{\gamma \dot{\mathbf{R}}}{m_{0}} .
$$

This third-order equation can be solved in time as a system of three first-order equations using a Runge-Kutta numerical integration procedure (Boyce and DiPrima, 1977). Since $\mathbf{W}=\dot{k}=\dot{h} / 2$, we find that Equation (7) is harmonic in $\mathbf{W}$, driven by a forcing function which is dependent on the production, $\dot{\mathbf{R}}$ :

$$
\ddot{\mathbf{W}}+\left(\frac{g_{z}+\dot{\mathbf{W}}}{k}\right) \mathbf{W}=\frac{\gamma \dot{\mathbf{R}}}{m_{0}} .
$$

Thus, if the avalanche is struck by an abrupt change in $\mathbf{R}$, caused by, say, a change in surface roughness, we expect an upward acceleration and velocity of the center of mass that may overshoot the equilibrium position, which is given by the reference flow height, $h_{0}, h>h_{0}$. This is demonstrated in Figure 2, where we apply two bursts of $\dot{\mathbf{R}}$ to an avalanche with initial flow height, $h_{0}=1 \mathrm{~m}$, and flow density, $\rho_{0}=300 \mathrm{~kg} \mathrm{~m}^{-3}$ (Fig. 2a-d). The magnitude of the overshoot defines the reduction in flow density (Fig. 2e). Such overshoots are visible at the heads of dense flowing avalanches, where flow densities are said to be 'dilute' and appear to fluctuate (Gauer and others, 2007a,b; Turnbull and McElwaine, 2007). Because gravity is always acting, the new flow state is not sustainable and is continually changing, unless, of course, the avalanche can maintain the production of $\mathbf{R}$. When $\mathbf{R}$ is constant, the density will oscillate around some value. At this value, $p=0$ and the normal stress, $N$, is equal to the weight of the avalanche, $N_{0}$ (Fig. 2f). The center of mass oscillates with the circular frequency

$$
\omega^{2}=\left(\frac{g_{z}+\dot{\mathbf{W}}}{k}\right) .
$$


Higher frequencies are associated with smaller heights and flatter slopes, as the vertical gravity component, $g_{z}$, increases. Without random energy input, $\mathbf{R}$ will dissipate and the mass will collapse to the reference height, $h_{0}$; the density will increase to $\rho_{0}$.

\section{CONCLUSIONS}

A conclusion we draw from our analysis is that dispersive pressures in an open volume cannot be of long duration. Bagnold (1954) showed that shearing increases the pressure in a closed volume. He found that as long as the granular volume is sheared, the dispersive pressure remains. In a shallow avalanche, being a system with an open boundary, the dispersive pressure increases the height (center of mass). The normal pressure oscillates around the hydrostatic pressure. This result has been found in many experiments (Bartelt and others, 2007; Platzer and others, 2007). Excursions from the hydrostatic pressure (dispersive pressure) alter the flow density of the avalanche. These changes in density can be maintained over long flow periods, if the production of random energy is in balance with its decay, $\dot{\mathbf{R}}=0$; that is, $\mathbf{R}$ is constant. This can easily occur on steep and even moderately inclined slopes. On flat slopes the dissipation of $\mathbf{R}$ will overcome the production and we expect to see an increase in flow density and shear resistance and therefore a decrease in avalanche speed.

Both the normal pressure and the flow height can be measured in small-scale chute experiments with granular materials (Bartelt and others, 2007) or large chute experiments with snow (Platzer and others, 2007). However, in granular experiments it is difficult to discern how changes in flow height affect the flow velocity. Equation (7) provides us with a tool to separate bulk changes in the normal pressure caused by the mass flux from changes induced by perpendicular accelerations and the associated changes in density. Because the mean velocity can likewise be measured, with high-speed cameras (Schaefer and others, 2010) or optical velocity sensors (Kern and others, 2009), it might be possible in the near future to quantify how perpendicular accelerations influence the velocity of the flow. Important relationships could be derived from experiments that describe how these accelerations alter the frictional shear stress, which determines the speed of the avalanche.

We derived Equation (7) with respect to the center of mass of a flow segment. Equation (7) can therefore be coupled with depth-averaged mass and momentum equations for avalanche flow (Savage and Hutter, 1989, 1991). The thirdorder equation can be decomposed into three first-order equations for $k, \dot{k}$ and $\ddot{k}$. By including the convective accelerations of $k, \dot{k}$ and $\ddot{k}$, a system of partial differential equations results which will allow depth-averaged solution methods to track changes in avalanche density. This would have great utility in practical problems, especially mitigation problems involving flow heights and pressures. Whether a simple coefficient, $\gamma$, describing the conversion of the random energy flux to mechanical (potential) energy, is sufficient, can only be determined when the full system of partial differential equations is solved, which will hopefully be in the near future.

\section{REFERENCES}

Bagnold, R.A. 1954. Experiments on a gravity-free dispersion of large solid spheres in a Newtonian fluid under shear. Proc. $R$. Soc. London, Ser. A, 225(1160), 49-63.

Bartelt, P. and O. Buser. 2011. Dispersive pressure and velocity fluctuations in avalanches. Reply to comment by K. Kelfoun and T. Davies on 'A random kinetic energy model for rock avalanches: eight case studies'. J. Geophys. Res., 116(F1), F01015. (10.1029/2010JF001956.)

Bartelt, P. and B.W. McArdell. 2009. Granulometric investigations of snow avalanches. J. Glaciol., 55(193), 829-833.

Bartelt, P., O. Buser and K. Platzer. 2006. Fluctuation-dissipation relations for granular snow avalanches. J. Glaciol., 52(179), 631-643.

Bartelt, P., O. Buser and K. Platzer. 2007. Starving avalanches: frictional mechanisms at the tails of finite-sized mass movements. Geophys. Res. Lett., 34(20), L20407. (10.1029/2007GL031352.)

Boyce, W.E. and R.C. DiPrima. 1977. Elementary differential equations and boundary value problems. New York, Wiley.

Buser, O. and P. Bartelt. 2009. Production and decay of random kinetic energy in granular snow avalanches. J. Glaciol., 55(189), 3-12.

Christen, M., J. Kowalski and P. Bartelt. 2010. RAMMS: numerical simulation of dense snow avalanches in three-dimensional terrain. Cold Reg. Sci. Technol., 63(1-2), 1-14.

Gauer, P. and 7 others. 2007a. On full-scale avalanche measurements at the Ryggfonn test site, Norway. Cold Reg. Sci. Technol., 49(1), 39-53.

Gauer, P., M. Kern, K. Kristensen, K. Lied, L. Rammer and H. Schreiber. 2007b. On pulsed Doppler radar measurements of avalanches and their implication to avalanche dynamics. Cold Reg. Sci. Technol., 50(1-3), 55-71.

Gubler, H. 1987. Measurements and modelling of snow avalanche speeds. IAHS Publ. 162 (Symposium at Davos 1986 - Avalanche Formation, Movement and Effects), 405-420.

Issler, D. and P. Gauer. 2008. Exploring the significance of the fluidized flow regime for avalanche hazard mapping. Ann. Glaciol., 49, 193-198.

Kern, M., P. Bartelt, B. Sovilla and O. Buser. 2009. Measured shear rates in large dry and wet snow avalanches. J. Glaciol., 55(190), 327-338.

Naaim, M., F. Naaim-Bouvet, T. Faug and A. Bouchet. 2004. Dense snow avalanche modeling: flow, erosion, deposition and obstacle effects. Cold Reg. Sci. Technol., 39(2-3), 193-204.

Norem, H., F. Irgens and B. Schieldrop. 1987. A continuum model for calculating snow avalanche velocities. IAHS Publ. 162 (Symposium at Davos 1986 - Avalanche Formation, Movement and Effects), 363-379.

Platzer, K., P. Bartelt and M. Kern. 2007. Measurements of dense snow avalanche basal shear to normal stress ratios $(S / N)$. Geophys. Res. Lett., 34(7), L07501. (10.1029/2006GL028670.)

Salm, B. 1993. Flow, flow transition and runout distances of flowing avalanches. Ann. Glaciol., 18, 221-226.

Savage, S.B. and K. Hutter. 1989. The motion of a finite mass of granular material down a rough incline. J. Fluid Mech., 199, $177-215$

Savage, S.B. and K. Hutter. 1991. The dynamics of avalanches of granular materials from initiation to runout. Part I: analysis. Acta Mech., 86(1-4), 201-223.

Schaefer, M., L. Bugnion, M. Kern and P. Bartelt. 2010. Position dependent velocity profiles in granular avalanches. Granular Matter, 12(3), 327-336.

Turnbull, B. and J.N. McElwaine. 2007. A comparison of powdersnow avalanches at Vallée de la Sionne, Switzerland, with plume theories. J. Glaciol., 53(180), 30-40. 